\title{
Compact Wideband Frequency Reconfigurable Metamaterial Antenna Design
}

\author{
Adamu Y Iliyasu*, Mohamad Rijal Bin Hamid, Mohamad Kamal A Rahim, Murtala Aminu-Baba and \\ Mohd Fairus Bin Mohd Yusof
}

Advance RF \& Microwave Research Group, School of Electrical Engineering, Faculty of Engineering, Universiti Teknologi Malaysia, 81310 UTM Skudai, Johor, Malaysia.

${ }^{*}$ Corresponding author: alhaji080@kustwudil.edu.ng

\begin{abstract}
This paper presents the design of compact wideband frequency reconfigurable metamaterial (MTM) antenna. The design is based on the idea of obtaining single and multi-bands in wideband metamaterial antenna within the range of bandwidth. This is achieved by introducing capacitive slots which neutralize inductive properties and generate left handed capacitive parameter. The three series slots in the patch contribute for bandwidth enhancement while two PIN Diode Switches provide multi-bands operation. Computer Simulation Technology (CST) software is used to determine the operation and effectiveness of the proposed antenna. The approach has several notable merits which include improvement of spectrum utilization, minimize spectrum congestion, interference and provide bands selectivity. From the simulation results, it was found that, bandwidth was improved to $2.8 \mathrm{GHz}$ which is equivalent to $82 \%$ fractional bandwidth. Also, it can switch to seven different frequency bands of operation with only two number of switches. The realized peak gain is $2.44 \mathrm{dBi}$ and $3.15 \mathrm{dBi}$ at $2.4 \mathrm{GHz}$ and $5.0 \mathrm{GHz}$ respectively with average efficiency of $95 \%$. The antenna can be utilized for wireless communication and cognitive radio application.
\end{abstract}

Keywords: Bandwidth (BW), Computer Simulation Technology (CST), Frequency Reconfigurable, Metamaterial (MTM), Multiband (MB).

\section{INTRODUCTION}

Rapid development and demand of wireless communication necessitated to have miniaturized, wideband and high efficient antenna for multi functions operation in different applications. However, single and multiband antennas are highly needed within the certain wideband range to minimize spectrum congestion. Metamaterials are unnatural structures with properties not available in nature depending on the negative value of microscopic parameters permittivity and permeability for operation improvement [1]. MTM can be classified based on the sign of $\varepsilon$ and $\mu$ as double positive $(\varepsilon>0, \mu>0$, dielectric), epsilon negative $(\varepsilon<0, \mu>0$, plasma), double negative $(\varepsilon$ $<0, \mu<0$ not exist in nature), and mu negative $(\varepsilon>0, \mu$ $<0$, gyrostropic) [2]. Composite Right/Left Handed unit cell are modeled based on it four lump element circuit parameters like series capacitance $C_{L}$, shunt inductance $L_{L}$ which account for left handed propagation and series inductance $L_{R}$, shunt capacitance $C_{R}$ for right handed propagation. Equation (1), (2), (3) and (4) represent the series resonant frequency, shunt resonant frequency and two frequencies for right and left hand respectively [3].

$$
\begin{aligned}
& \omega_{\mathrm{se}}=\frac{1}{\sqrt{\mathrm{L}_{\mathrm{R}} \mathrm{C}_{\mathrm{L}}}} \\
& \omega_{\mathrm{sh}}=\frac{1}{\sqrt{\mathrm{C}_{\mathrm{R}} \mathrm{L}_{\mathrm{L}}}}
\end{aligned}
$$

$$
\begin{aligned}
& \omega_{\text {R.H }}=\frac{1}{\sqrt{\mathrm{L}_{\mathrm{R}} \mathrm{C}_{\mathrm{R}}}} \\
& \omega_{\text {.L.H }}=\frac{1}{\sqrt{\mathrm{L}_{\mathrm{L}} \mathrm{C}_{\mathrm{L}}}}
\end{aligned}
$$

Based on the lump element circuit parameters, low value of right handed capacitance and high value of left handed inductance result to low quality factor which lead to bandwidth enhancement as shown in equation (5) and (6) respectively [4].

$$
\begin{aligned}
& \mathrm{Q}=\frac{1}{\mathrm{G}} \sqrt{\frac{\mathrm{C}_{\mathrm{R}}}{\mathrm{L}_{\mathrm{L}}}} \\
& \mathrm{BW}=\mathrm{G} \sqrt{\frac{\mathrm{L}_{\mathrm{L}}}{\mathrm{C}_{\mathrm{R}}}}
\end{aligned}
$$

Reconfigurable antennas are class of antennas that have the capacity to select it operating parameters such as polarization, frequency, or radiation pattern to redistribute it current for frequency selectivity and reuse, they can be classified based on frequency, pattern or polarization [5].

There are several attempts for BW enhancement and reconfiguration by using MTM for high efficient and multi operation antennas. It was reported in [6], BW enhancement was achieved by originating and merge mode near the fundamental mode by utilizing CRLHTL parameters. Similarly, author in [7] also enhance the BW by inserting closed ring resonator. Author in [8] found certain differences with almost the same approach, 
suggesting that, multi frequency operation can be obtain from MTM antennas. Similar methodology was used by [9]. but results to wideband and multi-band by using ring resonators. Moreover, several antennas with multiband application were presented by [10], [11] and [12] utilizing metamaterial behavior. Frequency reconfigurable was applied on resonant antennas and achieved multiband by [13], [14] and [15]. In this paper, a compact frequency reconfigurable metamaterial antenna with multi function capability is designed and analyzed. The proposed antenna operates from the basic of [6] with the same overall dimension but with some differences in physical parameters. The fundamental objective of this design is bandwidth enhancement and reconfiguration to improve spectrum utilization and band selectivity due to multi-bands obtained with less number of switches in $\mathrm{ON}$ state. The simulation work was done by using computer simulation technology (CST MWS) software.

\subsection{ANTENNA DESIGN}

Figure 1 shows the physical geometry of the proposed antenna. As mentioned earlier, this antenna was designed based on the basic foundation of [6]. First, the ohm's shape slot was replaced with horizontal slot, and extend inductive strip $\boldsymbol{C}$ and $\boldsymbol{D}$ from $1 \mathrm{~mm}$ to $5.3 \mathrm{~mm}$ and $2.38 \mathrm{~mm}$ respectively for high value of left-handed inductance. Position of the horizontal slots for bandwidth were determined by taking the parametric studies along $\mathrm{X}$ axis.

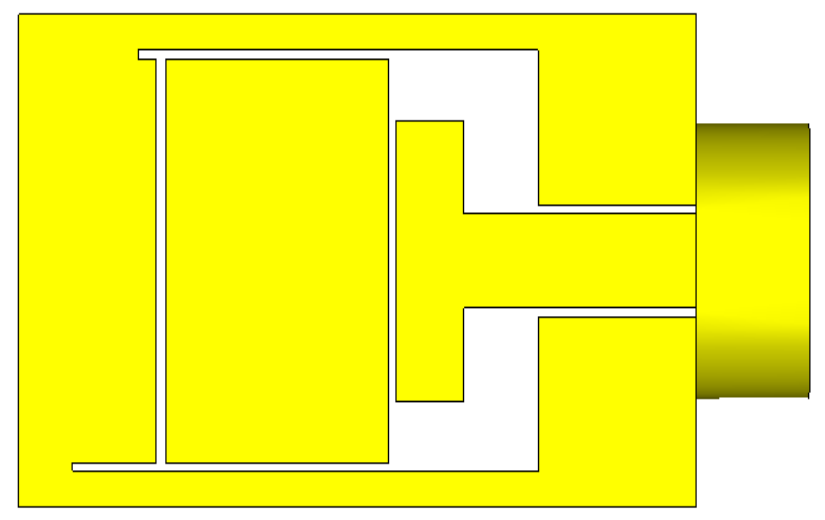

Figure 1. Proposed Antenna

Figure 2 shows the final version of proposed structure with three horizontal slots in the top patch and two PIN diode switches at two different positions. Two switches were positioned based on the behavior of the current distribution of antenna at $2.4 \mathrm{GHz}$. This is to achieve frequency reconfiguration process. The structure has overall dimension of 16.80 by $30.0 \mathrm{~mm}^{2}$ with the following dimensions in $m m, \mathbf{A}=\mathbf{3 0 . 0}, \mathbf{B}=\mathbf{1 6 . 8}, \mathbf{C}=\mathbf{5 . 3}$, $\mathrm{D}=2.38, \mathrm{E}=0.4, \mathrm{~F}=0.5, \mathrm{G}=0.5, \mathrm{H}=0.3, \mathrm{I}=1.4$, and $\mathrm{J}=\mathbf{6 . 5}$.
A

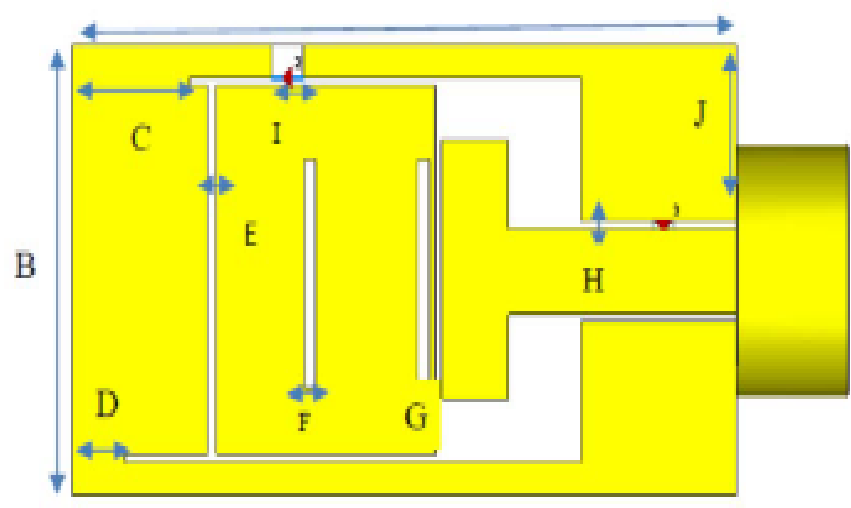

Figure 2. Geometrical Configuration of Proposed Antenna

Figure 3 shows the schematic diagram of the proposed antenna indicating the actual arrangement of switches for frequency reconfiguration.

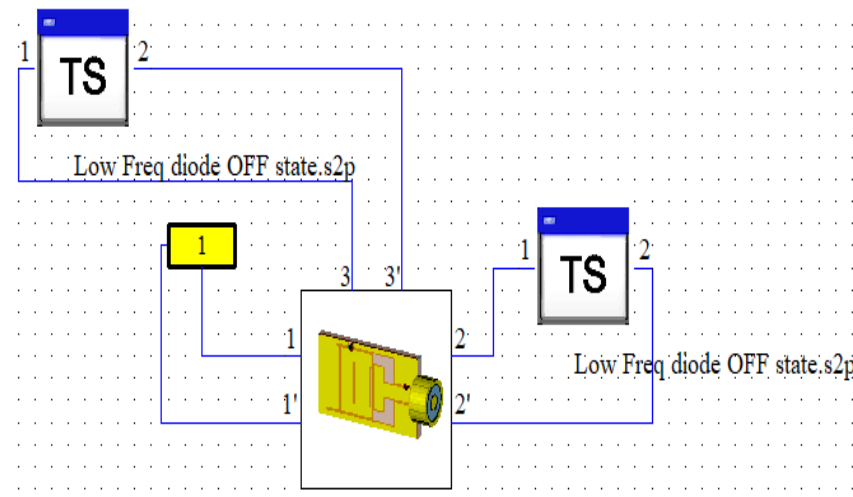

Figure 3. Schematic Diagram of Proposed Antenna

\subsection{RESULTS AND DISCUSSION}

Figure 4 shows the parametric results of the proposed antenna for the major horizontal slot at the top patch with thickness $\mathbf{E}$ along x-axis in four different position of $L_{n}$ This is to locate the appropriate position for the bandwidth enhancement. Bandwidth with the range of 2.2 to $4.3 \mathrm{GHz}$ was obtained at $L_{n}=5.8 \mathrm{~mm}$.

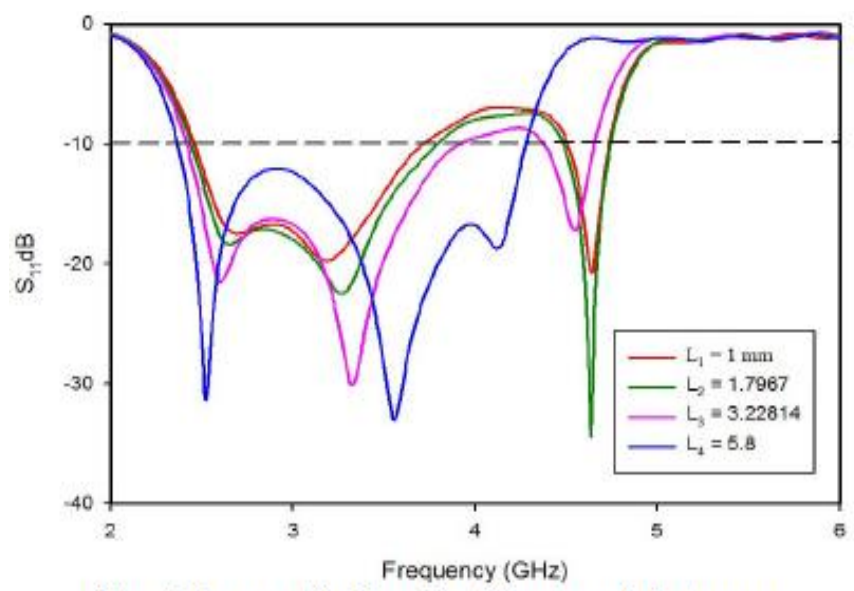

Figure 4. Parametric Results of Proposed Antenna 
Figure 5 shows the improved bandwidth with bandwidth range of 2.3 to $5.1 \mathrm{GHz}$ by introducing two more capacitive slots in the top patch with improve matching at $2.3 \mathrm{GHz}$ and $5.0 \mathrm{GHz}$ operating bands. This improvement is as a result of low quality factor which lead to bandwidth enhancement as shows in equation (5) and (6).

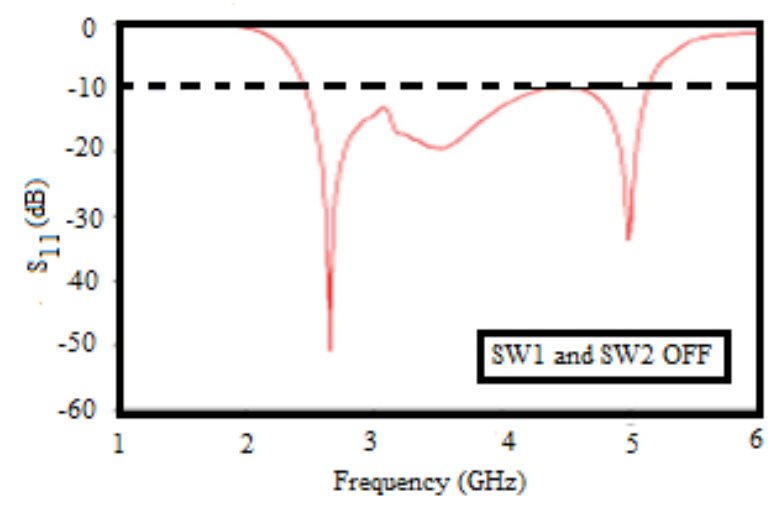

Figure 5. Enhanced Bandwidth of Proposed Antenna

Table 1.1 presents the summary of the switch configurations, operating bands and their status.

Table 1. Table caption, centre

\begin{tabular}{|c|c|c|c|}
\hline SW 1 & SW 2 & Status & Bands (GHz) \\
\hline OFF & OFF & Wideband & 2.4 and 5.1 \\
\hline ON & OFF & Dual-band & 1.3 and 3.0 \\
\hline OFF & ON & $\begin{array}{c}\text { Multi- } \\
\text { band }\end{array}$ & $2.1,3.0$ and 5.0 \\
\hline ON & ON & Dual-band & 1.2 and 3.0 \\
\hline
\end{tabular}

From the switch configuration in table 1.1 above, the following results were obtained. When both SW1 and SW2 are OFF, wide-band with range of (2.3-5.1) $\mathrm{GHz}$ was obtained. For SW1 ON, SW2 OFF a single-band within the range of wide- band at $3.0 \mathrm{GHz}$ and extra band at $1.3 \mathrm{GHz}$, also for SW1 OFF and SW2 ON, dual-bands at $3.0 \mathrm{GHz}$ and $5.0 \mathrm{GHz}$ within the range of wide-band are obtained with extra $2.1 \mathrm{GHz}$ band outside the wideband, Finally, the $3.0 \mathrm{GHz}$ o p e r a t i n g band was obtained within the wide-band and $1.2 \mathrm{GHz}$ outside wideband for both SW1 and SW2 in ON state. Figures 6 (a), (b), (c) and (d) presents the results for all switch configuration.

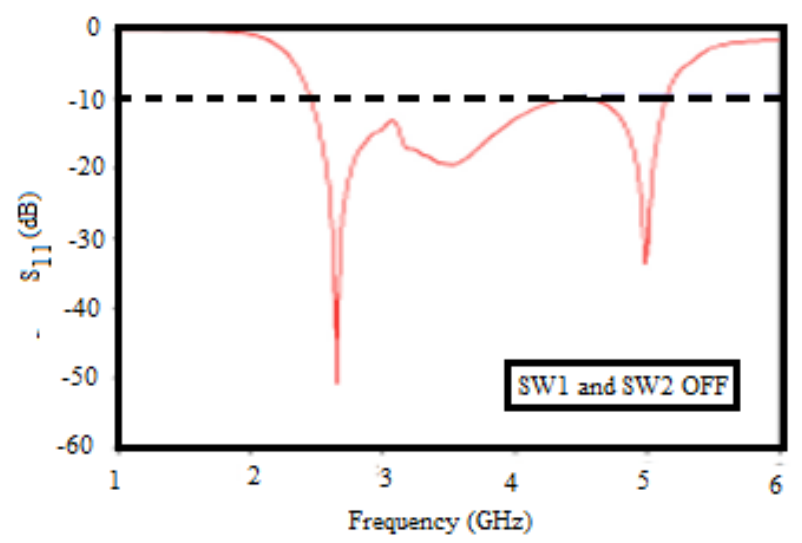

(a)

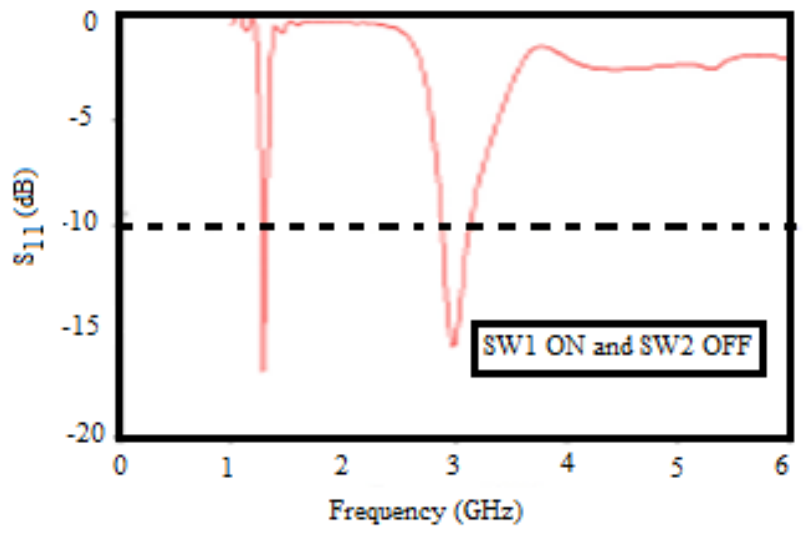

(b)

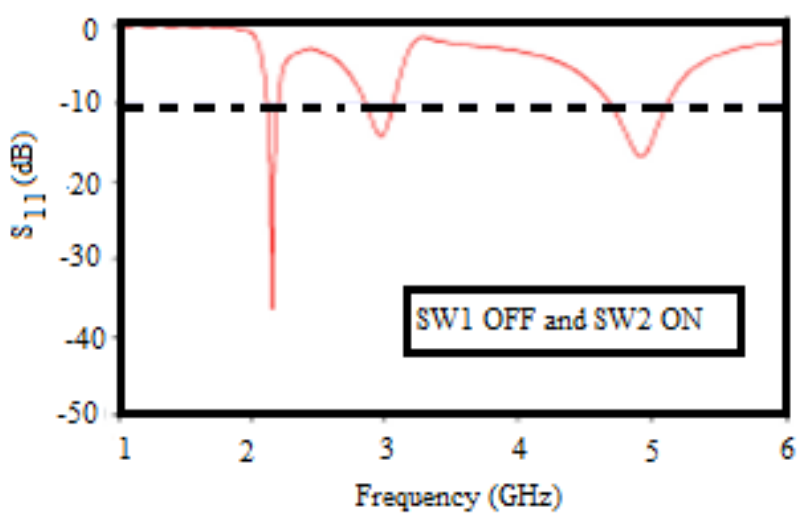

(c)

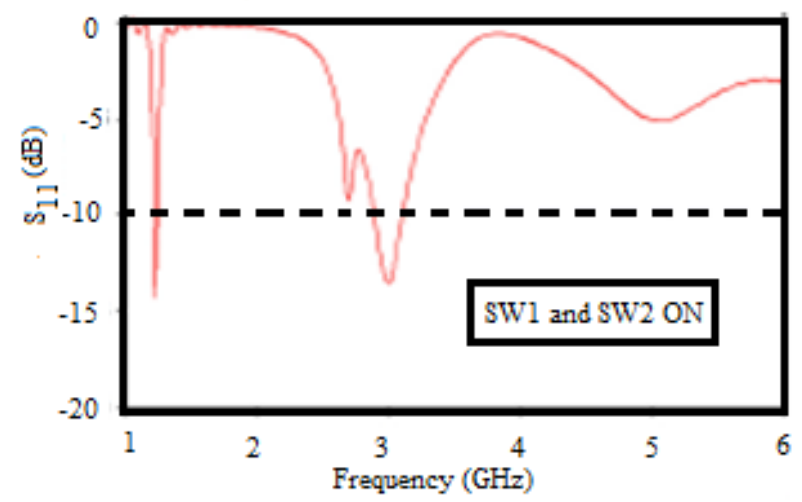

(d)

Figure 6. Switch Configuration Results (a) SW1 and SW2 OFF (b) SW1 ON SW2 OFF (c) SW1 OFF SW2 ON (d) SW1 and SW2 ON 
Figure. 7 shows the radiation pattern of the proposed antenna at $2.4 \mathrm{GHz}$ and $5.0 \mathrm{GHz}$ with realized peak gain of $2.44 \mathrm{dBi}$ and $3.15 \mathrm{dBi}$ respectively which show good omnidirectional at $5.0 \mathrm{GHz}$.

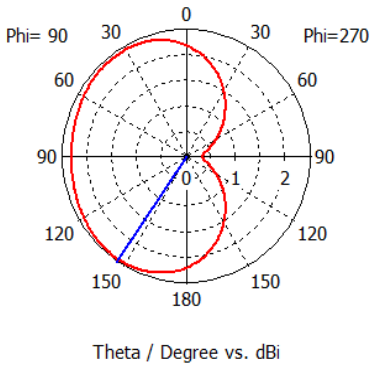

(a)

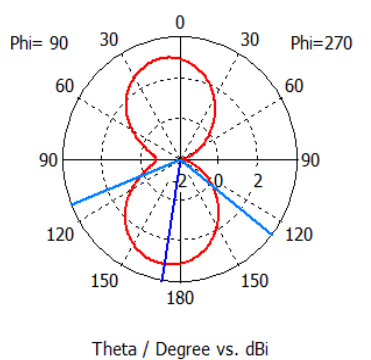

(b)
Figure 7. Radiation Pattern at (a) $2.4 \mathrm{GHz}$ (b) 5.0 $\mathrm{GHz}$

Table 2 presents the summary of the comparison between results obtain from this work and previous work. The summary showed that, there is significant improvement in terms of bandwidth, gain, efficiency and available operating bands.

Table 2. Table caption, centre

\begin{tabular}{|c|c|c|c|c|c|}
\hline Parameter & $\begin{array}{c}\text { This } \\
\text { Work }\end{array}$ & {$[4]$} & {$[5]$} & {$[6]$} & {$[7]$} \\
\hline FBW & 76 & 54.3 & 48.5 & - & - \\
\hline Efficiency & 95 & 92.8 & 97 & 90 & 93 \\
\hline Peak Gain & 2.0 & 2.36 & 2.97 & 1.95 & Low \\
\hline Bands & $\begin{array}{c}\text { WB, } \\
\text { SB and } \\
\text { MB }\end{array}$ & $\begin{array}{c}\text { WB } \\
\text { only }\end{array}$ & $\begin{array}{c}\text { WB } \\
\text { Only }\end{array}$ & $\begin{array}{c}\text { MB } \\
\text { and } \\
\text { SB }\end{array}$ & $\begin{array}{c}\text { WB } \\
\text { and SB }\end{array}$ \\
\hline
\end{tabular}

\section{CONCLUSION}

The main objective was to design metamaterial antenna with bandwidth enhancement and obtain single and multi bands within the range of bandwidth by frequency reconfiguration technique. The investigation proved that, it is possible to enhance the bandwidth of antenna by increasing the value of left-handed capacitance and achieved frequency reconfiguration by introducing inductive strip or capacitive slot to obtain multi-bands. The simulation work shown that, bandwidth was improved from $54.3 \%$ to $76 \%$ by introducing three capacitive horizontal slots in top patch. It also provides single band and multiband at seven different operating bands as shown in table 1.2 by reconfiguration technique. Further work can be done to improve the matching of the results obtained, and fabrication need to be done for measurement and comparison of the simulation and experimental result.

\section{REFERENCES}

[1] R. Rajkumar and K. Usha Kiran, "A Metamaterial Inspired Compact Open Split Ring Resonator Antenna for Multiband Operation," Wirel. Pers. Commun., vol. 97, no. 1, pp. 951-965, 2017.

[2] F. Bilotti and L. Sevgi, "Metamaterials: Definitions , Properties, Applications, and FDTD-Based Modeling and Simulation," Int. J. RF Microw. Comput. Eng., vol. Vol. 000, 2012.

[3] A. Lai, S. Member, K. M. K. H. Leong, and T. Itoh, "Infinite Wavelength Resonant Antennas With Monopolar Radiation Pattern Based on Periodic Structures," IEEENTRANSACTIONS ANTENNAS Propag., vol. 55, no. 3, pp. 868-876, 2007.

[4] T. Jang, J. Choi, and S. Lim, "Compact coplanar waveguide (CPW)-fed zeroth-order resonant antennas with extended bandwidth and high efficiency on vialess single layer," IEEE Trans. Antennas Propag., vol. 59, no. 2, pp. 363-372, 2011.

[5] J. Costantine, Y. Tawk, S. E. Barbin, and C. G. Christodoulou, "Reconfigurable antennas: Design and applications," Proc. IEEE, vol. 103, no. 3, pp. 424-437, 2015.

[6] A. Artemenko et al., "A Compact CPW-Fed Wideband Metamaterial Antenna Using $\Omega$ - Shaped Interdigital Capacitor for Mobile Applications," MICROWAVE AND OPTICAL TECHNOLOGY LETTERS vol. 57, no. 11, pp. 2558-2562, 2015.

[7] A. Gupta, S. K. Sharma, and R. K. Chaudhary, "A Compact CPW-fed Metamaterial Antenna for High Efficiency and Wideband Applications," pp. 3-6.

[8] A. B. Jagadeesan, A. Alphones, M. F. Karim, and L. C. Ong, "Metamaterial based reconfigurable multiband antenna," IEEE Antennas Propag. Soc. AP-S Int. Symp. vol. 2015-Octob, pp. 2389-2390, 2015.

[9] S. K. Sharma and R. K. Chaudhary, "Frequency reconfigurable mobile antenna with multi-band characteristics based on metamaterials," Proc. 2015 Int. Conf. Microw. Photonics, ICMAP 2015, pp. 8-9, 2016.

[10] H. Huang, Y. Liu, S. Zhang, and S. Gong, "Multiband Metamaterial-loaded Monopole Antenna for WLAN / WiMAX Applications," vol. 1225, no. 61372001, 2014.

[11] M. A. Abdalla and A. A. Ibrahim, "Multi-band MetaMaterial Antenna with Asymmetric Coplanar Strip-Fed Structure," pp. 631-632, 2015.

[12] A. Kumar, "Microstrip Patch Antenna Loaded with Metamaterial for Multiband Applications," pp. 43-47, 2016.

[13] S. S. Behera and S. Sahu, "Frequency Reconfigurable Antenna Inspired by Metamaterial for WLAN and WiMAX Application," no. I, pp. 442-446, 2014.

[14] M. I. H. M. R. I. Faruque and M. T. I. M. T. Ali, "Design and analysis of coupled-resonator reconfigurable antenna," Appl. Phys. A, vol. 122, no. 1, pp. 1-4, 2016.

[15] M. A. R. M. R. I. F. M. T. Islam, "Circularly split-ringresonator-based frequency-reconfigurable antenna," Appl. Phys. A, vol. 123, no. 1, pp. 1-6, 2017. 